\title{
Evaluation of antimicrobial susceptibilities and virulence factors of Staphylococcus aureus strains isolated from community-acquired and health-care associated pediatric infections
}

\author{
Adem Karbuz ${ }^{1}$, Zeynep Ceren Karahan², Bilge Aldemir-Kocabaş ${ }^{1}$, Alper Tekeli², Halil \\ Özdemir ${ }^{1}$, Haluk Güriz ${ }^{3}$, Refik Gökdemir ${ }^{3}$, Erdal İnce ${ }^{1}$, Ergin Çiftçi ${ }^{1}$ \\ Departments of ${ }^{1}$ Pediatric Infectious Diseases, ${ }^{2}$ Clinical and Basic Microbiology, ${ }^{3}$ Basic Microbiology, Ankara University \\ Faculty of Medicine, Ankara, Turkey. E-mail:karbuzadem@hotmail.com \\ Received: 21st February 2017, Accepted: 28th March 2017
}

SUMMARY: Karbuz A, Karahan ZC, Aldemir-Kocabaș B, Tekeli A, Özdemir H, Güriz H, Gökdemir R, İnce E, Çiftçi E. Evaluation of antimicrobial susceptibilities and virulence factors of Staphylococcus aureus strains isolated from community-acquired and health-care associated pediatric infections. Turk J Pediatr 2017; 59: 395-403.

The aim of this study was to investigate the enterotoxins and Panton-Valentine leukocidin $(P V L)$ gene as virulence factor, identification if antimicrobial sensitivity patterns, agr (accessory gene regulator) types and sequence types and in resistant cases to obtain SCCmec (staphylococcal cassette chromosome mec) gene types which will be helpful to decide empirical therapy and future health politics for $S$. aureus species. Total of 150 isolates of $S$. aureus were isolated from the cultures of the child patients in January 2011 and December 2012. In this study, the penicillin resistance was observed as $93.8 \%$. PVL and mecA was detected positive in $8.7 \%$ and in $6 \%$ of all $S$. aureus strains, respectively. Two MRSA (methicillin resistant S.aureus) strains were detected as SCCmec type III and SCCmec type V and five MRSA strains were detected as SCCmec type IV. SET-I and SET-G were the most common detected enterotoxins. In both community-associated and healthcare-associated MRSA strains, agr type 1 was detected most commonly. The most common sequence types were ST737 in 13 patients than ST22 in eight patients and ST121 in six patients. This study highlights a necessity to review the cause of small changes in the structural genes in order to determine whether it is a cause or outcome; community-acquired and healthcare associated strains overlap.

Key words: pediatric infections, pulsed field gel electrophoresis profile, Staphylococcus aureus, virulence factors.

S. aureus is an important pathogen causing both health-care associated (HCA) and communityacquired (CA) infections. Its prevalence is increasing particularly in pediatric intensive care units. Molecular typing data have revealed that there are a few methicillin resistant Staphylococcus aureus (MRSA) clones responsible for spreading MRSA infections in different regions of the world. ${ }^{1}$

Methicillin resistance in staphylococci depends on the production of penicillin-binding protein (PBP)-2a, which has a low affinity to all betalactam antibiotics and can maintain cell wall synthesis in the presence of these antibiotics.
PBP-2a is encoded by a gene named mec $A$ which is found on a mobile genetic element named the "staphylococcal cassette chromosome mec (SCCmec)".1,2 Up to date, 11 different SCCmec types have been identified. Although HCAMRSA strains more frequently carry the type-I, -II or -III and CA-MRSA isolates carry type-IV, -V or -VI SCCmec genes, this is not a rule.

Various virulence factors such as staphylococcal enterotoxins (ET), toxic shock syndrome toxin (TSST)-1, exfoliative toxins (ETA), and leukocidins including hemolysins and PantonValentine leukocidin (PVL) are responsible for the different clinical syndromes associated 
with S. aurues infections. The expression of many virulence factors of $S$. aureus is controlled by accessory gene regulator (agr) locus encoding a two-component signaling pathway. ${ }^{3}$

It is quite difficult to determine the role of each staphylococcal virulence factor in the pathogenesis of invasive infections although it has been shown that PVL production is particularly associated with the development of furuncles, skin abscesses, and severe necrotic tissue infections. ${ }^{1,4}$

In this study, we aimed to evaluate the antimicrobial susceptibilities, agr and SCCmec types and virulence factors of HCA- and CA-S. aureus strains isolated as etiological infectious agents in children, and to establish the multilocus sequence types (MLST) of representative strains showing different pulsed field gel electrophoresis (PFGE) profiles. With the data obtained, we also aimed to contribute to the molecular epidemiology of S. aureus strains of younger age group in our country, and guide the clinicians in the empirical treatment of coexisting infections.

\section{Material and Methods}

The study was conducted on 150 S. aureus strains isolated from pediatric in- or outpatients in the Department of Pediatrics between January 2011 and December 2012.

Patient groups: All strains were isolated from non-duplicate patients who were younger than 18 years. The patients were divided into community-acquired and healthcare-associated infection groups according to the definition of CDC in 2000.5

Detection of antibiotic susceptibilities: KirbyBauer disc diffusion test was performed in line with the criteria of Clinical Laboratory Standards Institute (CLSI) to determine the antimicrobial susceptibilities of the isolates. ${ }^{6}$

Molecular analyses: DNA was extracted by using "GeneMATRIX Bacterial Genomic DNA Extraction Kit (EUR ${ }_{X}$, Polland) according to the manufacturer's instructions. Presence of mecA and PVL genes were evaluated by PCR analyses as described previously. 7,8 SCCmec and agr types and enterotoxin genes of the isolates were determined by multiplex PCR methods as described in the literature. ${ }^{9-11}$

Pulsed field gel electrophoresis (PFGE): Canadian PFGE protocol was used with minor modifications. ${ }^{12}$ Lysostaphine concentration was doubled, and incubation period in Proteinase-K buffer was extended to two hours. The principles determined by Tenover et al. ${ }^{13}$ were adopted in the evaluation of genotypic relationship among isolates. DNA patterns and genetic relatedness of the isolates were analyzed by using the Gene Directory Programme (Syngene, UK). The similarity index was determined by using the Dice coefficient with $1.5 \%$ band tolerance. Cluster analysis of the isolates was performed by the unweighted pair-group method on the basis of arithmetic averages (UPGMA).

Multilocus sequence typing (MLST): Representative isolates of each pulsotype were randomly chosen for MLST analysis. ${ }^{14}$

Table I. Distribution of Isolates According to Their Sites of Infection.

\begin{tabular}{lccc}
\hline Diagnosis & CA & HCA & Total \\
\hline Skin and soft tissue infections, n (\%) & $45(64.2)$ & $32(40.0)$ & $77(51.3)$ \\
Eye and ear infections, n (\%) & $16(22.8)$ & $3(3.7)$ & $19(12.6)$ \\
Bacteremia-sepsis-endocarditis, n (\%) & $4(5.7)$ & $14(17.5)$ & $18(12.0)$ \\
Catheter-related infections, n (\%) & - & $17(21.2)$ & $17(11.3)$ \\
Bone and joint infections, n (\%) & $2(2.8)$ & $3(3.7)$ & $5(3.3)$ \\
Meningitis, n (\%) & - & $4(5.0)$ & $4(2.6)$ \\
Urinary tract infections, n (\%) & $2(2.8)$ & $2(2.5)$ & $4(2.6)$ \\
Ventilator-associated pneumonia, n (\%) & - & $2(2.5)$ & $2(1.3)$ \\
Peritonitis, n (\%) & - & $2(2.5)$ & $2(1.3)$ \\
Pneumonia and empyema, n (\%) & $1(1.4)$ & $1(1.2)$ & $2(1.3)$ \\
\hline Total, n & 70 & 80 & 150 \\
\hline
\end{tabular}

CA: community acquired, HCA: health-care associated 
Table II. Antimicrobial Resistance Rates of S. aureus Strains in Community Acquired and Health-care Associated Infections.

\begin{tabular}{|c|c|c|c|c|c|c|}
\hline \multirow[b]{2}{*}{ Antimicrobial drug } & \multicolumn{2}{|c|}{ CA (\% resistance) } & \multicolumn{2}{|c|}{ HCA (\% resistance) } & \multicolumn{2}{|c|}{ Total (\% resistance) } \\
\hline & I & $\mathrm{R}$ & I & $\mathrm{R}$ & I & $\mathrm{R}$ \\
\hline Penicillin & - & 91.2 & - & 96.2 & - & 93.8 \\
\hline Ampicillin-sulbactam & 1.5 & 7.5 & & 5.4 & 0.7 & 6.5 \\
\hline Cefoxitin & - & 7.1 & - & 5.1 & - & 6 \\
\hline Erythromycin & 10.1 & 7.2 & 1.3 & 6.7 & 5.5 & 6.9 \\
\hline Rifampicin & - & - & - & 5.2 & - & 2.8 \\
\hline Clindamycin & 8.8 & 5.8 & - & 5.3 & 4.1 & 5.5 \\
\hline Gentamycin/netilmicin & - & - & 1.5 & 1.5 & 0.7 & 0.7 \\
\hline Ciprofloxacin & 4.6 & - & 7.8 & 7.1 & 6.3 & 2.8 \\
\hline TMP-SMX & - & 1.4 & 1.2 & 5 & 0.6 & 3.3 \\
\hline Tetracycline & 1.5 & 1.5 & - & 12.3 & 0.7 & 7.2 \\
\hline
\end{tabular}

CA: community acquired, HCA: health-care associated, I: intermediate, R: resistant, TMP-SMX: trimethoprimsulphamethoxazole

\section{Statistical analysis}

Statistical analysis was performed at the Department of Biostatistics, by using the SPSS 17.0 statistical software. Descriptive statistics were demonstrated as mean for normally distributed variables, median for abnormally distributed variables, and the number of patients and percentage (\%) for nominal values. The significance of the difference between the groups with regard to the means was investigated with Student's t-test and the significance of difference with regard to the median was analyzed with Mann-Whitney U test. A p value of $<0.05$ was accepted to be statistically significant.

The ethical approval was obtained from the Ethics Committee of Medical Faculty of University (13.06.2011/32-265). The written consent form was taken from all the patients.

\section{Results}

The study included a total of 150 patients, 54 in 2011 and 96 in 2012. Of the patients, 70 had CA-S. aureus infection and 80 had HCA-S. aureus infection; $58.7 \%$ of patients were male $(n=88)$ and the median age was 19.5 months (3 days-18 years). The distribution of isolates according to their sites of infection are given in Table I, with skin and soft tissue infections being the most prevalent ones in both CA- and HCA- groups.

Antimicrobial resistance rates of the isolates are given in Table II. In $6 \%(n=9)$ of the strains, mecA positivity was detected. All except one of the mecA-positive strains were phenotypically found to be methicillin-resistant. Only one phenotypically resistant strain was $m e c A$-negative. Two of the mecA-positive MRSA strains was SCCmec type-III, two were SCCmec type-V and five were SCCmec type IV. Of the mecA-positive strains, four $(44.4 \%)$ were CAS. aureus.

PVL positivity was observed in $8.7 \%(n=13)$ of all S. aureus strains. The most commonly determined enterotoxin genes were of enterotoxins I and G. No enterotoxin gene was found in 62 of the $S$. aureus strains. The rest of the strains carried at least one enterotoxin gene. Thirty-nine $(26 \%)$ of the isolates carried more than one enterotoxin gene. Enterotoxin genes, $P V L$-positivity, mecA-positivity, and agr types of CA- and HCA-S. aureus strains are shown in Table III.

Seventy-four different pulsotypes were determined by PFGE; 61 of these strains were divided into 26 groups which included at least 2 and at most 4 strains (Fig. 1).

Sequence types were determined for $89 \mathrm{~S}$. aureus strains (Table IV). Twenty-nine different sequence types were detected in $50 \mathrm{CA}-$ and in 39 HCA-S. aureus strains.

\section{Discussion}

Community acquired MRSA frequency has been 
increasing and there is a closer relationship between CA-MRSA and invasive skin and soft tissue infections. ${ }^{15,16}$ In our study, the rate of MRSA was found to be $6 \%$ in the pediatric patient group. Of these strains, $2.6 \%$ were CA infections and $3.4 \%$ were HCA infections. While skin and soft tissue infections (abscess, impetigo, and omphalitis) and conjunctivitis were detected in patients with CA-MRSA, patients with HCA-MRSA had more serious clinical conditions such as endocarditis, sepsis, post-operative meningitis, surgical wound site infection, and urinary tract infection. The mean ages of patients with CA-MRSA and HCA-MRSA were 32 months and 85.6 months, respectively. According to the results of our study, MRSA does not seem to be a major problem for Turkey, at least in the pediatric patients. However, it should be kept in mind that MRSA has a tendency to cause more serious and invasive infections.

The studies revealed that TMP-SMX is still a preferable antibiotic for empirical treatment of skin and soft tissue infections associated with MRSA strains in most regions of the world. ${ }^{17}$ While all of the CA-MRSA strains were susceptible to ciprofloxacin, rifampicin, erythromycin, and clindamycin, 25\% were resistant to TMP-SMX in our study. In HCAMRSA strains, resistance rates were found as $40 \%$ for rifampicin, $40 \%$ for TMP-SMX, $\% 20$ for erythromycin and $20 \%$ for clindamycin. Although it is a small study population, the detection of TMP-SMX resistance in $25 \%$ of patients with CA-MRSA infections suggests that empirical use of TMP-SMX in patients

Table III. Enterotoxins, PVL, agr Types, Positivity of mecA for Community Acquired and Health-care Associated S. aureus Strains.

\begin{tabular}{|c|c|c|c|c|c|c|}
\hline \multirow[b]{2}{*}{ Characteristic } & \multicolumn{2}{|c|}{$\begin{array}{c}\text { MRSA [mecA }(+)] \\
(\mathrm{n}=9)\end{array}$} & \multicolumn{2}{|c|}{$\begin{array}{c}\text { MSSA [mecA }(-)] \\
(n=141)\end{array}$} & \multicolumn{2}{|c|}{$\begin{array}{c}\text { Total } \\
(\mathrm{n}=150)\end{array}$} \\
\hline & $\begin{array}{c}\mathrm{CA} \\
(\mathrm{n}=4)\end{array}$ & $\mathrm{HCA}(\mathrm{n}=5)$ & $\begin{array}{c}\text { CA } \\
(n=66)\end{array}$ & $\begin{array}{c}\text { HCA } \\
(n=75)\end{array}$ & $\begin{array}{c}\mathrm{CA} \\
(\mathrm{n}=70)\end{array}$ & $\begin{array}{c}\mathrm{HCA} \\
(\mathrm{n}=80)\end{array}$ \\
\hline agr type 1 & 3 & 5 & 27 & 41 & 30 & 46 \\
\hline agr type 2 & - & - & 10 & 14 & 10 & 14 \\
\hline agr type 3 & 1 & - & 19 & 14 & 20 & 14 \\
\hline agr type 4 & - & - & 8 & 4 & 8 & 4 \\
\hline agr locus ND & - & - & 2 & 2 & 2 & 2 \\
\hline SCCmec type I & - & - & - & - & - & - \\
\hline SCCmec type II & - & - & - & - & - & - \\
\hline SCCmec type III & - & 2 & - & - & - & 2 \\
\hline SCCmec type IV & 3 & 2 & - & - & 3 & 2 \\
\hline SCCmec type V & 1 & 1 & - & - & 1 & 1 \\
\hline PVL & - & - & 9 & 4 & 9 & 4 \\
\hline ET-A & - & 2 & 1 & 4 & 1 & 6 \\
\hline ET-C & - & - & 4 & 2 & 4 & 2 \\
\hline ET-E & - & - & 1 & 5 & 1 & 5 \\
\hline ET-G & - & - & 20 & 17 & 20 & 17 \\
\hline ET-I & 1 & 2 & 36 & 38 & 37 & 40 \\
\hline ET-G + ET-I & 1 & 1 & 14 & 13 & 15 & 14 \\
\hline ET-C + ET-G + ET-I & 1 & - & 3 & 2 & 4 & 2 \\
\hline ET-A + ET-I & - & - & - & 2 & - & 2 \\
\hline ET-E + ET-J & - & - & - & 1 & - & 1 \\
\hline ET-A + ET-E + ET-I & - & - & - & 1 & - & 1 \\
\hline
\end{tabular}

agr: accessory gene regulator, CA: community acquired, ET : enterotoxin, HCA: health-care associated, ND: not detected, PVL:Panton-Valentine leucocidin, SCC:staphylococcal cassette chromosome, MRSA: methicillin resistant Staphylococcus aureus, MSSA: methicillin susceptible Staphylococcus aureus 
with a suspect of MRSA infection will be inappropriate. Our study shows that the use of penicillinase-resistant beta-lactam antibiotics and clindamycin will be a more appropriate choice for empirical treatment of CA-methicillin sensitive $S$. aureus infections.

In studies conducted in the USA, PVL positivity was reported at a rate of $74-100 \%$ in MRSA strains and $9-46 \%$ in MSSA strains depending on the regions where data were collected. ${ }^{18}$
Furthermore, a relationship was found between SCCmec types of MRSA strains and PVL positivity. While PVL positivity was below $5 \%$ in MRSA strains having SCCmec type- I-III, it was between $40 \%$ and $90 \%$ in MRSA strains having SCCmec type-IV. ${ }^{19}$ The most common sequence types determined in PVL positive CA-MRSA strains were ST8 in the USA, ST80 in Europe, ST30 and ST59 in the Asian-Pacific area. ${ }^{20-23}$

Table IV. The Detailed Distribution of the Types of Sequences of S. aureus Strains According to Groups.

\begin{tabular}{|c|c|c|c|c|c|c|c|}
\hline \multirow{2}{*}{$\begin{array}{l}\text { Sequence types } \\
\text { (ST) }\end{array}$} & \multicolumn{2}{|c|}{ CA } & \multicolumn{2}{|c|}{ HCA } & \multirow[b]{2}{*}{ Total } & \multirow[b]{2}{*}{ PVL } & \multirow[b]{2}{*}{ mecA } \\
\hline & MSSA & MRSA & MSSA & MRSA & & & \\
\hline ST5 & 3 & - & 2 & - & 5 & & - \\
\hline ST6 & 1 & & 1 & - & 2 & & - \\
\hline ST7 & 1 & - & 1 & - & 2 & & - \\
\hline ST8 & 3 & & 1 & - & 4 & & - \\
\hline ST9 & 1 & - & 2 & - & 3 & & - \\
\hline ST10 & 1 & - & - & - & 1 & & - \\
\hline ST12 & 1 & - & - & - & 1 & & - \\
\hline ST15 & 3 & - & 4 & - & 7 & 2 & - \\
\hline ST22 & 5 & 1 & 2 & - & 8 & 2 & 1 \\
\hline ST25 & 2 & - & - & - & 2 & & - \\
\hline ST27 & 1 & - & - & - & 1 & & - \\
\hline ST30 & 5 & - & 1 & - & 6 & 2 & - \\
\hline ST34 & - & - & 3 & - & 3 & 1 & - \\
\hline ST45 & - & - & 2 & - & 2 & & - \\
\hline ST46 & 2 & - & - & - & 2 & & - \\
\hline ST88 & - & - & 1 & - & 1 & 1 & - \\
\hline ST97 & - & - & 4 & - & 4 & & - \\
\hline ST121 & 4 & - & 2 & - & 6 & 3 & - \\
\hline ST188 & 1 & - & 2 & - & 3 & & - \\
\hline ST199 & - & - & 1 & - & 1 & & - \\
\hline ST239 & - & - & 1 & 2 & 3 & & 2 \\
\hline ST291 & 2 & - & - & - & 2 & & - \\
\hline ST398 & 1 & - & - & - & 1 & & - \\
\hline ST489 & 1 & - & - & - & 1 & & - \\
\hline ST509 & - & 1 & 1 & - & 2 & & 1 \\
\hline ST737 & 5 & 2 & 5 & 1 & 13 & & 3 \\
\hline ST837 & 1 & - & - & - & 1 & & - \\
\hline ST1021 & 1 & - & - & - & 1 & & - \\
\hline ST1708 & 1 & - & - & - & 1 & 1 & - \\
\hline Total & 46 & 4 & 36 & 3 & 89 & 12 & 7 \\
\hline
\end{tabular}

Data is presented in numbers. CA: community acquired, HCA: health-care associated, PVL:panton-valentine leucocidin, MRSA: methicillin resistant Staphylococcus aureus, MSSA: methicillin susceptible Staphylococcus aureus 
PVL positivity is not so common in our country as in most European countries. In a study performed in Turkey, 242 S. aureus strains isolated from skin and soft tissue infections were evaluated for the presence of $P V L$ genes, and PVL positivity was detected in $9.1 \%$ of the strains, all of which were MSSA; $63.6 \%$ of the PVL-positive strains were isolated from CA- and $36.4 \%$ were from HCA infections. PVL positivity was not detected in any of the MRSA strains. ${ }^{24}$ In another study conducted in Turkey, PVL-positivity was reported to be $<10 \%$ for MSSA and $<3 \%$ for MRSA. ${ }^{25}$ In our study, PVL positivity was found in $8.7 \%$ of the strains, all of which were MSSA. PVL-positive $S$. aureus was found in $69.2 \%$ of CA-MSSA strains and in $30.8 \%$ of HCA-MSSA strains. Of the PVL producing strains, $77 \%$ were isolated from patients with skin and soft tissue infections, $7.7 \%$ from bone and joint infections, $7.7 \%$ from patients diagnosed with pneumonia-empyema, and $7.7 \%$ from patients with catheter-related infection. Of the MRSA strains, seven were SCCmec types-IV and -V. None of them carried the PVL gene. PVL negativity particularly in the CA-MRSA strains can be explained with their sequence types (ST737, ST509 and ST22). As in other studies, a stronger relationship was found between PVL positivity and skin and soft tissue infections in our study. ${ }^{26}$

When mecA positivity is regarded as the goldstandard, the sensitivity and specificity of oxacillin/cefoxitin disc diffusion method for determining methicillin resistance have been reported to be $100 \%$ and $89 \%$, respectively. 27 The rates of sensitivity and specificity were found to be $88.8 \%$ and $99.2 \%$ for oxacillin/cefoxitin disc diffusion method in our study. One, mecAnegative strain was phenotypically methicillinresistant, and one mecA-positive strain was phenotypically MSSA. Misidentification of one mecA-positive strains as MSSA might have resulted from heterogeneous appearance of methicillin resistance, or technical problems such as the use of inappropriately stored betalactam antibiotic disks, incubation conditions, and amount of inoculum. The determination of mecA-negative strain as MRSA may be due to point mutations in penicillin binding proteins, excess production of penicillinase, or a decrease in the binging affinity of methicillin. ${ }^{28}$

In a study carried out in Taiwan, $91.9 \%$ of the isolates were agr type-1, $7.8 \%$ type-2, $0.2 \%$ type-3, and $0.1 \%$ type $4 .{ }^{29}$ In another study conducted in England, 26\% of HCA-MSSA isolates represented agr group 2. ${ }^{30}$ In another study, while agr type 3 was observed to be associated with non-invasive diseases, agr type 1 was associated with invasive diseases such as bacteremia. ${ }^{31}$ In our study, $50.7 \%$ were found to be agr type 1, 16\% type 2, $22.7 \%$ type 3 , and $8 \%$ type 4 . The most common agr type in CA and HCA infections was type 1 ( $42.9 \%$ and $57.5 \%$, respectively). While $a g r$ types 1 and 2 were most frequently detected in HCA-infections, agr types 3 and 4 were more prevalent in community acquired infections ( $\mathrm{p}>0.05)$. In MRSA strains, agr types 1 and 3 were detected in $88.8 \%$ and $12.2 \%$ of the strains. agr type 1 was more prominent in strains isolated from invasive infections such as meningitis and bacterial endocarditis and in bone-joint infections, compared to other types. On the other hand, agr type 4 was more frequently found in skin and soft tissue infections. Considering infection types and antibiotic susceptibility profiles, it is seen that these data are generally similar to the literature all over the world.

In a study that was performed in Germany, $25.6 \%$ of the $S$. aureus strains were found to be negative for enterotoxin genes. Enterotoxins with at least two and more combinations were observed in $63.9 \%$ of strains and single enterotoxin gene was found at in $10.5 \%$ of the strains. ${ }^{32}$ In another study, enterotoxins $G$ and I were more frequently detected and a statistically significant difference was found between MRSA and MSSA strains $(77 \%$ for MRSA and $49 \%$ for MSSA). The coexistence of enterotoxins $\mathrm{G}$ and $\mathrm{I}$, as well as D and J, was also remarkable ( $17 \%$ for MRSA, $8 \%$ for MSSA) in another study. ${ }^{11}$ In our study, enterotoxins $\mathrm{B}, \mathrm{D}, \mathrm{H}$ and TSST-1 were not detected in any of the strains. No enterotoxin was found in 62 isolates $(41.3 \%)$. At least one, at most three coexistences of enterotoxin genes were observed. Enterotoxin I presence was most frequent $(51.3 \%)$, followed by $\mathrm{G}(24.6 \%)$, A (4.6\%), C (4\%), E (4\%), and J (0.66\%).

The results of PFGE typing in our study, grouped the strains in 74 different pulsotypes. Detection of so many different pulsotypes may be due to the long study time of two years, 
no occurrence of an outbreak in our hospital during this period, collection of the samples from different clinics, and the higher number of patients with community acquired infections.

There is not much data about molecular characterization of CA-MRSA strains in our country, especially in the pediatric population. This is the first study covering the detection of enterotoxin and PVL genes, as well as SCCmec types, agr types, and PFGE profiles with MLST analysis in the pediatric patient group in Turkey. In a study which evaluated nine possibly HAMRSA strains in Turkey between 2003 and 2004, MLST analysis was performed and their sequence types were found to be ST239.33 In our study, MLST analysis was performed in 100 strains. Based on the allelic profiles, sequence types could be determined in 89 strains. 29 different sequence types were found and the most common ones were ST737 (14,6 $\%)$, ST22 (8,9\%), ST15 (7,8 \%), ST30 (6,7 $\%)$, ST121 (6,7 \%), and ST5 (5,6\%). While ST737, ST22, ST30, and ST121 were the most common types in CA-strains, ST737, ST97, ST15, ST239, and ST34 were most prevalent in HCA-strains. There are not many reports on ST737 sequence type, which has one-allele difference with ST22. The frequency of ST737 strains was found to be $4 \%$ in S. aureus strains collected from different regions of our country between 2005 and $2007^{34}$, it was $14.6 \%$ in our study. Rapid spread of this strain both in the community and hospital environment is noteworthy. To investigate whether the pathogenicity of ST737 strain is different from others or not will reveal significant results in terms of preventive public health measures and empirical treatment. It was interesting to observe the presence of various sequence types within the same pulsotype in our study, which may reflect that even in the same PFGE group, different clones may exist (Fig. 1).

In conclusion, our study has provided important epidemiological data on $S$. aureus strains isolated from pediatric patients in Turkey. Although CA$S$. aureus infections were not a major problem in our study population; considering the process followed in different regions of the world, we assume that CA-infections would increase over time. PVL-positivity is still more prevalent among MSSA strains, although gaining the $\operatorname{mec} A$ gene among these populations may lead

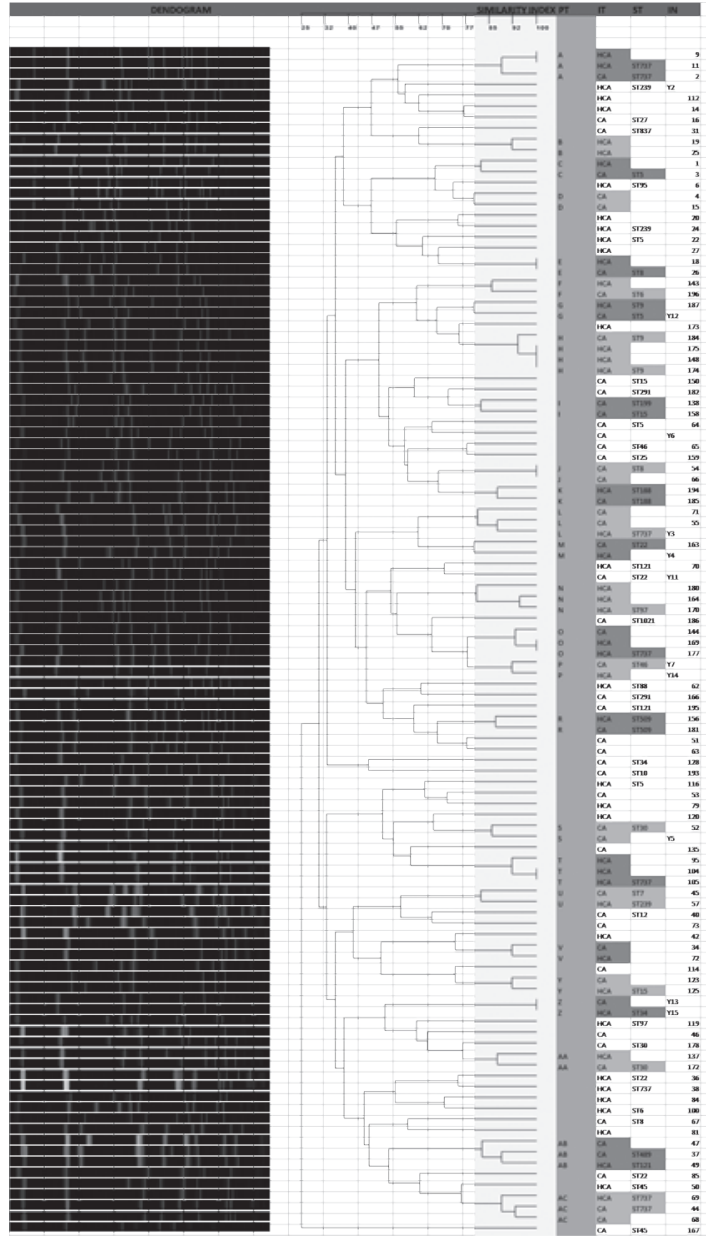

Fig. 1. Pulsed field gel electrophoresis (PFGE) pulsotypes and corresponding sequence types in $S$. aureus strains CA: community acquired, HCA: health-care associated, IN: isolate numbers, IT: infection types, PT: pulsotypes, ST: sequence types

to an increase in PVL-positive MRSA infections with higher morbidity and even mortality. Penicillinase-resistant beta-lactam antibiotics could still be used as the first choice in empirical treatment of pediatric patients with S. aureus infections, if they are not methicillinresistant. The rate of PVL positivity in Turkey was not found as high as other countries. When sequence types of both community and hospital acquired $S$. aureus strains were evaluated, the presence of interwoven strains was found to be consistent with the tendency recently reported across the world.

\section{Acknowledgement}

This research was supported by the University Research Fund (The project number is 12B3330002). 


\section{REFERENCES}

1. Lowell GS, Daum RS. Staphylococcus aureus. In: Long SS, Pickering LK, Prober CG (ed). Principles and Practise of Pediatric Infectious Diseases (3th ed). Philadelphia: Churchill Livingstone, 2008: 679-693.

2. Deurenberg RH, Stobbereingh EE. The evolution of Staphylococcus aureus. Infect Genet Evol 2008; 8: 747 763.

3. Tekeli A, Koyuncu E, Dolapçı İ, Akan AÖ, Karahan CZ. Molecular characteristics of methicillin-resistant Staphylococcus aureus strains isolated from blood cultures between 2002-2005 in Ankara University Hospital. Mikrobiyol Bul 2009; 43: 1-10.

4. Gillet Y, Issartel B, Vanhems P, et al. Association between Staphylococcus aureus strains carrying gene for Panton-Valentine leukocidin and highly lethal necrotising pneumonia in young immunocompetent patients. Lancet 2002; 359: 753-759.

5. Morrison MA, Hageman JC, Klevens RM. Case definition for community-associated methicillinresistant Staphylococcus aureus. J Hosp Infect 2006; 62: 241.

6. Clinical and Laboratory Standards Institute. Performance standards for antimicrobial susceptibility testing; 20th informational supplement. Document M100-S20. CLSI, Wayne, PA. 2012

7. Adaleti R, Nakipoglu Y, Karahan ZC, Tasdemir C, Kaya F. Comparison of polymerase chain reaction and conventional methods in detecting methicillin-resistant Staphylococcus aureus. J Infect Dev Ctries 2008; 2: 46-50.

8. Dufour P, Gillet Y, Bes M, et al. Community-acquired methicillin-resistant Staphylococcus aureus infections in France: Emergence of a single clone that produces Panton-Valentine leukocidin. Clin Infect Dis 2002; 35: 819-824.

9. Milheirico C, Oliveira DC, de Lencastre H. Multiplex PCR strategy for subtyping the staphylococcal cassette chromosome mec type IV in methicillin-resistant Staphylococcus aureus: 'SCCmec IV multiplex. J Antimicrob Chemother 2007; 60: 42-48.

10. Gilot P, Lina G, Cochard T, Poutrel B. Analysis of the genetic variability of genes encoding the RNA III-activating components agr and trap in a population of Staphylococcus aureus strains isolated from cows and mastitis. J Clin Microbiol 2002; 40: 4060-4067.

11. Sila J, Sauer P, Kolar M. Comparison of the prevalence of genes coding for enterotoxins, exfoliatins, PantonValentine leukocidin and TSST-1 between methicillinresistant and methicillin-susceptible isolates of Staphylococcus aureus at the University Hospital in Olomouc. Biomed Pap Med Fac Univ Palacky Olomouc Czech Repub 2009; 153: 215-218.

12. Mulvey MR, Chui L, Ismail J, et al. Canadian Committee for the Standardization of Molecular Methods. Development of a Canadian standardized protocol for subtyping methicillin-resistant Staphylococcus aureus using pulsed-field gel electrophoresis. J Clin Microbiol 2001; 39: 3481-3485.
13. Tenover FC, Arbeit RD, Goerıng RV, et al. Interpreting chromosomal DNA restriction patterns produced by pulsed-field gel electrophoresis: Criteria for bacterial strain typing. J Clin Microbiol 1995; 33: 2233-2239.

14. Enright MC, Day NP, Davies CE, Peacock SJ, Spratt BG. Multilocus sequence typing for characterization of methicillin-resistant and methicillin-susceptible clones of Staphylococcus aureus. J Clin Microbiol 2000; 38: 1008-1015.

15. Mera RM, Suaya JA, Amrine-Madsen $\mathrm{H}$, et al. Increasing role of Staphylococcus aureus and community-acquired methicillin-resistant Staphylococcus aureus infections in the United States: A 10-year trend of replacement and expansion. Microb Drug Resist 2011; 17: 321-328.

16. Como-Sabetti K, Harriman KH, Buck JM, Glennen A, Boxrud DJ, Lynfield R. Community-associated methicillin-resistant Staphylococcus aureus: Trends in case and isolate characteristics from six years of prospective surveillance. Public Health Rep 2009; 124: 427-435.

17. Britton PN, Andresen DN. Paediatric communityassociated Staphylococcus aureus: A retrospective cohort study. J Paediatr Child Health 2013; 49:754-759.

18. Ritz N, Curtis N. The role of Panton-Valentine leukocidin in Staphylococcus aureus musculoskeletal infections in children. Pediatr Infect Dis J 2012; 31: 514-518.

19. Deurenberg RH, Vink C, Kalenic S, Friedrich AW, Bruggeman CA, Stobberingh EE. The molecular evolution of methicillin-resistant Staphylococcus aureus. Clin Microbiol Infect 2007; 13: 222-235.

20. Roberts JC, Krueger RL, Peak KK, Veguilla W, Cannons AC, Amuso PT. Community-associated methicillinresistant Staphylococcus aureus epidemic clone USA300 in isolates from Florida and Washington. J Clin Microbiol 2006; 44: 225-6.

21. Chen CJ, Unger C, Hoffmann W, Lindsay JA, Huang YC, Götz F. Characterization and comparison of 2 distinct epidemic community-associated methicillinresistant Staphylococcus aureus clones of ST59 lineage. PLoS One 2013; 8: e63210.

22. Song JH, Hsueh PR, Chung DR, et al. Spread of methicillin-resistant Staphylococcus aureus between the community and the hospitals in Asian countries: An ANSORP study. J Antimicrob Chemother 2011; 66:1061-1069.

23. Wannet WJ, Heck ME, Pluister GN, Spalburg E, van Santen MG, Huijsdans XW. Panton-Valentine leukocidin positive MRSA in 2003: The Dutch situation. Euro Surveill 2004; 9:28-29.

24. Demir T, Coplu N, Bayrak H, et al. Panton-Valentine leucocidin gene carriage among Staphylococcus aureus strains recovered from skin and soft tissue infections in Turkey. J Antimicrob Chemother 2012; 67: 837-840.

25. Karahan ZC, Tekeli A, Adaleti R, et al. Investigation of Panton-Valentine leukocidin genes and SCCmec types in clinical Staphylococcus aureus isolates from Turkey. Microb Drug Resist 2008; 14: 203-210.

26. Shallcross LJ, Fragaszy E, Johnson AM, Hayward AC. The role of the Panton-Valentine leucocidin toxin in staphylococcal disease: A systematic review and metaanalysis. Lancet Infect Dis 2013; 13: 43-54. 
27. Swenson JM, Williams PP, Killgore G, O'hara CM, Tenover FC. Performance of eight methods, including two new rapid methods, for detection of oxacillin resistance in a challenge set of Staphylococcus aureus organisms. J Clin Microbiol 2001; 39: 3785-3788.

28. Yoshida RK Kuwahara-Arai K, Baba T, Cui L, Richardson JF, Hiramatsu K. Physiological and molecular analysis of a mecA-negative Staphylococcus aureus clinical strain that expresses heterogeneous methicillin resistance. J Antimicrob Chemother 2003; 51: 247-255.

29. Ho CM, Hsueh PR, Liu CY, et al. Prevalence and accessory gene regulator (agr) analysis of vancomycinintermediate Staphylococcus aureus among methicillinresistant isolates in Taiwan--SMART program, 2003. Eur J Clin Microbiol Infect Dis 2010; 29: 383-389.

30. Moore PC, Lindsay JA. Genetic variation among hospital isolates of methicillin-sensitive Staphylococcus aureus: Evidence for horizontal transfer of virulence genes. J Clin Microbiol 2001; 39: 2760-2767.
31. Ben Ayed S, Boutiba-Ben Boubaker I, Samir E, Ben Redjeb S. Prevalence of agr specificity groups among methicillin resistant Staphylococcus aureus circulating at Charles Nicolle hospital of Tunis. Pathol Biol (Paris) 2006; 54: 435-438.

32. Becker K, Friedrich AW, Lubritz G, Weilert M, Peters G, Von Eiff C. Prevalence of genes encoding pyrogenic toxin superantigens and exfoliative toxins among strains of Staphylococcus aureus isolated from blood and nasal specimens. J Clin Microbiol 2003; 41: 1434-1439.

33. Ergon MC, Biçmen M, Gülay Z. Dokuz Eylül Üniversitesi Hastanesi'ndeki dominant metisiline dirençli Staphylococcus aureus suşunun moleküler yöntemlerle tiplendirilmesi. ANKEM Dergisi 2010; 24: 65-70.

34. Bozdoğan B, Yıldız O, Oryaşın E, et al. t030 is the most common spa type among methicillin-resistant Staphylococcus aureus strains isolated from Turkish hospitals. Mikrobiyol Bul 2013; 47: 571-581. 